\title{
075 AN INTERVENTION TRIAL FOR PEOPLE WITH EPILEPSY ATTENDING A\&E
}

Leone Ridsdale, ${ }^{1}$ Paul McCrone, ${ }^{1}$ Myfanwy Morgan, ${ }^{1}$ Paul Seed, ${ }^{1}$ Laura Goldstein, ${ }^{1}$ Adam Noble ${ }^{2}$. ${ }^{1}$ King's College, London; ${ }^{2}$ University of Liverpool

\subsection{6/jnnp-2014-309236.75}

Background Some people with epilepsy (PWE) make frequent emergency department (ED) visits, with up 50\% being admitted. No UK studies have examined interventions for them. We aimed to test whether an epilepsy nurse specialist (ENS) might reduce $\mathrm{ED}$ attendance.

Methods 85 adults with epilepsy were recruited, 41 receiving treatment-as-usual (TAU), and 44 PWE offered 2 one-to-one sessions with an ENS. Participants completed questionnaires at baseline, 6- and 12-months' follow-up.

Results Sixty-nine (81\%) participants completed follow-up. Over $40 \%$ were of non-white ethnicity, which predicted significantly higher service use and total costs. The intervention group had no significant relative reduction in ED visits at 12 months. Factors most predictive of subsequent ED visits were patients' baseline feelings of stigmatization due to epilepsy, and low confidence in managing epilepsy. Intervention participants spent less time as inpatients, so their average service cost was lower (adjusted difference $£ 558,95 \%$ CI, $-£ 2409, £ 648$ ).

Conclusions The intervention did not lead to a reduction in ED use, but did not cost more, partly because those receiving the intervention had shorter hospital admissions. Future interventions might focus more on patients' perceptions of stigma, and on their confidence in managing epilepsy. ED visits might, thereby, be reduced and efficiency-savings generated. 